\title{
TECNÓPOLIS EN CONTEXTOS COMPLEJOS: RESILIENCIA TECNOLÓGICA PARA EL DESARROLLO ECONÓMICO VENEZOLANO
}

\author{
Alfredo Emilio Ordóñez López ${ }^{1 *}$ \\ ${ }^{1}$ Universidad Central de Venezuela, Facultad de Ciencias Económicas y Sociales, Caracas-Venezuela \\ *Autor para correspondencia: e-mail: alfordonez@gmail.com
}

Recibido: 2018/01/03

Aprobado: 2018/05/06

DOI: https://doi.org/10.26621/XIV18.2018.06.A09.PUCESI.2550.6684

\section{RESUMEN}

El inicio del siglo XXI se caracteriza por la gran desigualdad en la riqueza de las naciones y la brecha industrial y tecnológica que distancia a las naciones del desarrollo económico. Ante esta visión, se refuerza la pertinencia de la búsqueda de modelos que permitan a las naciones equilibrar estos factores y generar resiliencia tecnológica ante la dinámica global. De tal manera, se necesitan capacidades elevadas de tecnología endógena para diferenciarse de los productos y/o servicios que ofrecen otras naciones. Es por ello que el principal objetivo del presente documento es analizar la capacidad que podría generar la creación de tecnópolis como sistema de alta resiliencia para la economía venezolana, frente a las tendencias de la economía global. Por tal sentido, la investigación propone, inicialmente, realizar un ajuste a la propuesta de Hartmut Bossel, tomando en cuenta la academia como un subsistema esencial para evaluar el desempeño económico, así como la incorporación de nuevos indicadores como la producción tecnológica, libre movilidad de fuerza de trabajo y cohesión social para los orientadores de este sistema, entendiendo que la dinámica y la evolución del sistema debe ir ajustándose a los contextos que se presentan en el mundo.

Palabras claves: Tecnópolis, resiliencia, tecnología, brecha tecnológica, industrialización.

\begin{abstract}
The beginning of the 21 st century is characterized by the huge inequality among nations regarding their wealth and the industrial and technological gaps that place countries away from economic development. This vision of inequality and technological gaps reinforces the need for searching models that allow nations balance these factors and generate resilience in face of global dynamics. Therefore, it is necessary to have high capacities of endogenous technology to differentiate themselves from other nation's products and/or services. The main objective of this document is to analyze the capacity to build Technopolis, as systems of high resilience for Venezuelan economy in face of global economic trends. In this sense, this investigation initially poses some adjustments to Hartmut Bossel's proposal, taking into account the Academy as an essential subsystem to evaluate economic performance, as well as the incorporation of new indicators such as technological production, workforce free mobility, social cohesion for the system guiders, thus understanding that its dynamic and evolution have to be adjusted according to different world contexts.
\end{abstract}

Keywords: Technopolis, resilience, technology, technological gap, industrialization 


\section{INTRODUCCIÓN}

Uno de los aspectos más determinantes en el escenario internacional es la productividad de los países para mantener el equilibrio político y económico del sistema. El aumento de las demandas internas y externas de cada país, obliga a los gobiernos a buscar estrategias que permitan satisfacer las necesidades y evitar altos grados de desigualdad en la sociedad. Sin embargo, esta situación se hace cada día más compleja. El deseado equilibrio se presenta menos alcanzable para los países en vías de desarrollo en vista de que la brecha tecnológica, cultural, religiosa, entre otros factores, inciden profundamente en sus estructuras productivas.

Países como Venezuela, con una clara dependencia económica de la industria petrolera, se caracterizan por ser economías vulnerables, lo que le ha generado la conformación de instituciones débiles y poco resilientes para generar cambios en sus estructuras productivas. Para Douglass North (1993) las instituciones son las reglas originadas en una sociedad que, a su vez, se transforman en las mismas limitaciones que dan forma a la interacción humana, por lo que se pueden admitir en las estructuras política, social y económica.

Un aspecto importante de North (1993) es que reconoce que las instituciones son una creación humana, por lo que las instituciones evolucionan y son alteradas por los mismos humanos. Si se concibe que la principal función de las instituciones en la sociedad sea reducir las incertidumbres estableciendo una estructura estable en el proceso de intercambio, se podría considerar la pertinencia de activar la resiliencia en las economías como la venezolana, para que éstas puedan ser más productivas ante la dinámica de la economía global. Y entre las alternativas, la tecnópolis sería una estrategia ideal para hacer frente a la dinámica de la economía mundial.

En tal sentido, el principal objetivo de este trabajo de investigación es analizar la capacidad que podría generar la creación de tecnópolis, como sistema de alta resiliencia para la economía venezolana frente a las tendencias de la economía global. La investigación tendrá como meta proponer indicadores que permitan conocer los niveles de complejidad y la capacidad de resiliencia de la estructura económica venezolana, tomando como base los indicadores propuestos por Hartmut Bossel para el Desarrollo Sostenible.

Para tales efectos, la investigación se sustentará en una investigación científica, que de acuerdo a los lineamientos generales del proceso de conocimiento, se enmarcará en un diseño de investigación documental de naturaleza holística, pues la misma se clasifica como predictiva al considerar una propuesta que permita solucionar problemas y/o necesidades de tipo práctico. El nivel de investigación, será de carácter explicativo y transversal dada la importancia de describir las variables y analizar su incidencia e interacción en un momento especifico, sin manipulación por parte del investigador.

\section{¿Qué son las Tecnópolis?}

Las tecnópolis son formas específicas de concentración territorial de innovación tecnológica, con potencial para generar sinergia científica y productividad económica. De tal manera, que se crea un sistema de estructuras sociales, institucionales, organizativas, económicas y territoriales, que producen las condiciones para la generación continua de sinergias y su inversión en un proceso de producción que se origina a partir de esta capacidad sinérgica, tanto para las unidades de producción, que son parte de este medio innovador, como para el medio en su conjunto (Castells y Hall, 1994).

Las tipologías de las tecnópolis representan diversos sistemas de medios donde la generación de innovación tecnológica es fundamental. Se caracterizan por ser estructuras claramente definidas en una localidad territorial que pueda generar sinergia científica, teniendo como resultado una mayor productividad económica, no sólo como generadora de rentabilidad en capital sino, también, como generadora de conocimiento endógeno y sostenible en el tiempo.

Para Castells y Hall (1994) existe una gran variedad de estructuras que se le pueden denominar tecnópolis en virtud de la experiencia internacional, pues no todas son específicamente iguales, pero si presentan el mismo objeto: generar conocimiento tecnológico. En tal sentido, clasifican a las tecnópolis en cuatro tipos: 
I. Complejos industriales (Complejos Tecno-Industriales) de empresas de alta tecnología que han sido construidos sobre la base de un medio innovador. Estos complejos, relacionando la I+D y la fabricación, son los centros de mando del nuevo espacio de producción. Por ejemplo: Silicon Valley, EE.UU.

II. Ciudades de la Ciencia. Complejos de investigación estrictamente científicos, sin relación territorial directa con la fabricación. Tiene como principal objetivo alcanzar el mayor nivel de excelencia científica mediante la sinergia que se supone generan en su aislado medio científico. Por ejemplo: Kansai, Japón.

III. Parques tecnológicos. Aspiran a inducir un nuevo crecimiento industrial, en términos de empleo y producción, intentando atraer a empresas de producción de alta tecnología a un espacio privilegiado. Cuentan con la iniciativa y el apoyo gubernamental y se relacionan con las universidades. Por ejemplo: Hsinchu, Taiwan; Sofía Antípolis, Francia y Cambridge, U.K.

IV. Programa de Tecnópolis. Representan el diseño de programas complejos de tecnópolis como instrumentos del desarrollo regional y la descentralización industrial. Consiste literalmente en un conjunto completo de nuevos medios innovadores para transferir el poder y generar nuevas tecnologías comerciales, desde el corazón del país a lugares que anteriormente carecían de tal capacidad. Establecen estrategias múltiples concentrando institutos de investigación públicos y privados, promoviendo tecnologías híbridas, mejorando los laboratorios de las universidades locales mediante el establecimiento de centros tecnológicos, financiando proyectos conjuntos de I+D. Por ejemplo: Tecnópolis, Japón.

Las tecnópolis son complejos industriales con una alta capacidad de investigación y desarrollo, generadores de innovación y ajustados a las tendencias de la economía mundial. Dichas estructuras no se encuentran establecidas en Venezuela, a pesar de la existencia de los grandes centros científicos y de investigación que podrían considerarse como pequeñas ciudades científicas, tales como el Instituto Venezolano de Investigaciones Científicas (IVIC), Instituto de Estudios Avanzados (IDEA), Fundación Instituto de Ingeniería (FII), Instituto Tecnológico Venezolano del Petróleo (INTEVEP), entre otros.

\section{¿Cómo generar tecnología en Venezuela?}

En esencia, la tecnología surge de la creatividad, la intuición, la inteligencia y la prospectiva, y puede crearse en el interior de una organización o adquirirse de fuentes externas, en algunos casos de manera libre y gratuita y, en otros, mediante acuerdos comerciales (Solleiro y Castallon, 2008). Por lo que se relaciona directamente a un conjunto de conocimientos expresado en técnicas aplicables para una finalidad particular, ya sea de manera tangible o intangible.

De acuerdo con Stewart (1983), la tecnología a disposición de un país particular es el total de las técnicas que conoce (o que puede llegar a conocer sin gran dificultad) y que podría adquirir, mientras que la tecnología en uso, es el subconjunto de las técnicas que ha adquirido ya. Para Stewart (1983), el concepto de tecnología no se puede quedar en la creación de herramientas, maquinaria, técnicas, procesos y ciencia; también tiene el objetivo de beneficiar (a la sociedad, organizaciones, Estados, gobiernos, individuos, etc) a través de las innovaciones, entendiendo está última como la generación de nuevos conocimientos.

El Estado como el principal rector del orden y cohesión social, deberá contemplar dentro de sus instituciones las reglas justas no sólo a nivel de seguridad jurídica, sino también para promover los resultados obtenidos por la plataforma tecnológica. En el enfoque de Sábato y Botana (1970) el vértice - gubernamental "tiene como objetivo el de formular o implementar políticas en el ámbito científico-tecnológico; ello requiere la capacidad para realizar una acción deliberada en este campo, para formular un cuerpo de doctrina, de principios y de estrategia capaz de fijar metas posibles, cuyo logro depende de una serie de decisiones políticas, de la asignación de recursos y de la programación científico-tecnológica" (p. 6). 
En el caso de las Universidades, serán responsables del proceso de formación del talento humano de alto nivel, mediante la concientización no sólo de los problemas de la sociedad, sino del cómo solucionarlo mediante la generación propia de herramientas que permitan atender las dificultades locales, nacionales y regionales. El proceso de concientización en el talento humano determina la aptitud del receptor del conocimiento para plantearse los problemas a enfrentar, y ello es, a base de la tecnología que comprende una serie de técnicas asociadas a un conjunto de características que van más allá de la naturaleza de un producto físico o del uso de los recursos - maquinaria, las mismas se fundamentan en la capacidad de generar, desarrollar, innovar, fomentar y promover estrategias de desarrollo conjuntas, que incidan de forma efectiva en los problemas comunes.

Cada una de las características, antes señaladas, resultan importantes para establecer la posibilidad y/o la conveniencia de la adopción de una técnica particular y, por ello, nacen las siguientes incógnitas:

1. ¿Cómo lograr esa evaluación?

2. ¿Quién implementa la tecnología seleccionada?

3. ¿Cómo garantizar el éxito de la tecnología aplicada?

4. ¿Qué fundamentos e indicadores podrá desempeñar esa tecnología?

En general, la región latinoamericana se ha convertido en importadora de tecnología, que ha generado una marcada tendencia a la incorporación de productos foráneos a la sociedad, promoviendo así cambios en sus estructuras de consumo y valores culturales. De tal manera que para modificar o eliminar la dependencia tecnológica foránea y reducir el grado de las importaciones de tecnología, se hace indispensable la conformación de un espacio generador de conocimiento (tecnológia) que permita la investigación y desarrollo de forma integral; y ello es posible en las tecnópolis, siendo estas estructuras capaces de lograr una gestión del conocimiento producir y controlar la información desarrollada.

Figura 1. Mecanismos de identificación de la tecnología a nivel nacional

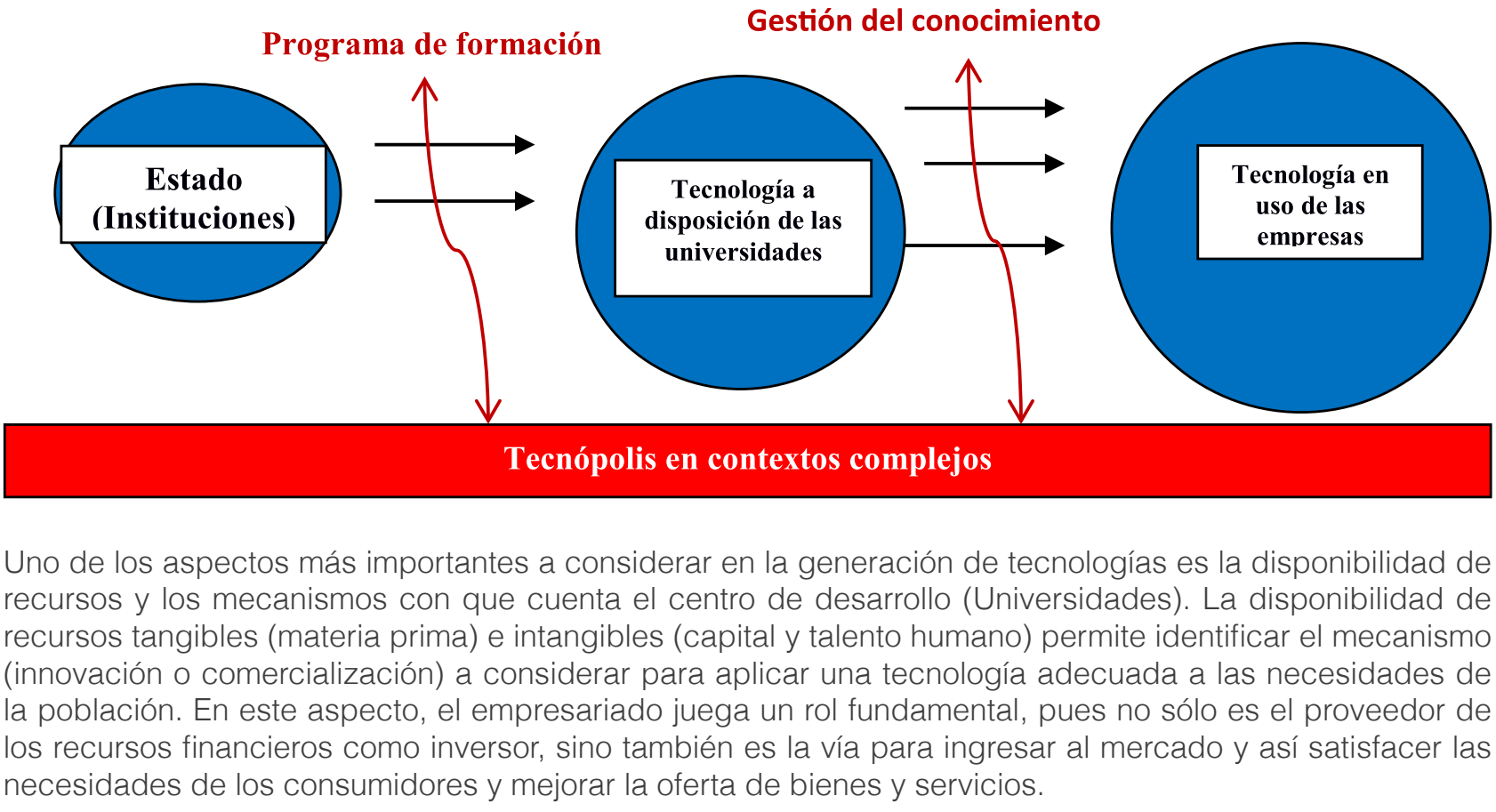

AXIOMA - Revista Científica de Investigación, Docencia y Proyección Social Enero-junio 2018 Número 18, pp 93-101. 
Sin embargo, la dinámica de las actuales políticas públicas implementadas para modificar ese ciclo vicioso de las importaciones de tecnología, se centran sólo en el uso de la tecnología extranjera como punto de despegue de la tecnología nacional, y no en la implementación de políticas que se enmarcan dentro del desarrollo endógeno de la tecnología nacional como opción, frente a la tecnología extranjera, generándose así una noción de la causación circular y acumulativa negativa. Gunnar Myrdal (1979) resalta un ejemplo de causación circular positiva de la siguiente manera: "es obvio que una relación circular integrada por menos pobreza, más alimentos, una salud mejor y una capacidad mayor para el trabajo, sostendría un proceso acumulativo positivo en vez de negativo" (p. 23).

En tal sentido, la vía para superar estos esquemas de dependencia tecnológica, es la especialización y desarrollo de centros tipo tecnópolis, para generar investigación y desarrollo, aunado a un proceso de transmisión, revisión (o adaptación) e implementación del conocimiento y el saber (know-how) para su uso productivo; lo que haría a la estructura económica venezolana más resiliente frente a la dinámica globalizadora del mundo e iniciaría un proceso de causación circular y acumulativo positivo.

Los beneficios que representarán las tecnópolis para activar la resiliencia económica de Venezuela, se materializarían en:

- Mayor rapidez en la aplicación de la tecnología y productividad con los recursos técnicos

- Mejores logros en el tiempo en los desarrollos tecnológicos y aplicaciones

- Menores costos para su aplicación y una mejor tecnología para mantener competitividad

- Soluciones técnicas a problemas existentes o necesidades futuras

- Diversificación del parque industrial, científico y tecnológico

- Crecimiento rápido de la base tecnológica

- Mayor capacidad de respuesta y de satisfacción a la población que más la necesita

\section{Las instituciones inclusivas y la resiliencia económica}

Acemoglu y Robinson (2003) sustentan su hipótesis sobre el porqué de los retrasos en la evolución de las naciones y localidades desde una perspectiva histórica. La mayor parte de la desigualdad del mundo apareció a partir de finales del siglo XVIII, poco después de la revolución industrial, y "... el éxito económico de los países difiere debido a las diferencias entre sus instituciones, a las reglas que influyen en cómo funciona la economía y a los incentivos que motivan a las personas" (p. 54).

Los países más desarrollados se caracterizan por contar con instituciones económicas inclusivas, las cuales posibilitan y fomentan la participación de la gran mayoría de las personas en actividades económicas, aprovechan mejor su talento y sus habilidades y permiten que cada individuo pueda elegir lo que desea. Las instituciones económicas inclusivas fomentan la actividad económica, el aumento de la productividad y la prosperidad económica.

En el caso contrario a las instituciones inclusivas, se presentan las instituciones económicas extractivas, estas son "... extractivas porque tienen como objetivo extraer rentas y riqueza de un subconjunto de la sociedad para beneficiar a un subconjunto distinto" (Acemoglu y Robinson, 2003: 56), las cuales se caracterizan en América Latina, África y hasta en Europa del Este, sociedades donde no se presentan igualdad de oportunidades ni un sistema legal imparcial.

Si se considera la premisa del deseo de cambio, la resiliencia tecnológica buscará mejores sendas de 
progreso por su enfoque inclusivo. He aquí el punto clave que juegan las tecnópolis, pues estas zonas locales industriales permitirán, en términos tecnológicos, promover y establecer mejores niveles de coordinación entre los agentes (Estado, Academia y Empresa), se incrementarán los niveles de innovación, logrando así avances tecnológicos para la satisfacción del mercado interno e incentivando la competitividad internacional, rompiendo así con el rentismo petrolero en el caso venezolano.

Tabla 1. Posible impacto de las Tecnópolis en las capacidades del sistema económico venezolano

\begin{tabular}{|c|c|c|}
\hline & $\begin{array}{c}\text { Tecnópolis - Instituciones inclusivas } \\
\text { Hay deseo de cambio }\end{array}$ & $\begin{array}{c}\text { Tecnópolis - Instituciones extractivas } \\
\text { No hay deseo de cambio }\end{array}$ \\
\hline & $\begin{array}{l}\text { Organización de los agentes económicos } \\
\text { (Estado-Empresa-Academia) }\end{array}$ & $\begin{array}{l}\text { - Asimetría entre las funciones de los } \\
\text { agentes }\end{array}$ \\
\hline$\checkmark$ & $\begin{array}{l}\text { Se inicia un proceso de desarrollo tecnológico } \\
\text { e industrial }\end{array}$ & $\begin{array}{l}\text { - Retraso tecnológico y obsolescencia } \\
\text { industrial }\end{array}$ \\
\hline$\checkmark$ & $\begin{array}{l}\text { Incrementa la competitividad de Venezuela en } \\
\text { el sistema económico mundial }\end{array}$ & $\begin{array}{ll}\text { - } & \text { Dependencia tecnológica y de las } \\
\text { importaciones }\end{array}$ \\
\hline
\end{tabular}

Con base a la metodología que establecen Bossel (1999) y Castellano (2013), para entender las capacidades sistémicas, al explicar que el sistema social está dividido en los subsistemas individuo, sociedad, gobierno, economía, infraestructura y naturaleza; se ha considerado limitar el campo de estudio exclusivamente a la esfera tecnológica, ajustar los orientadores a la creación de tecnópolis y proponer nuevos indicadores, manteniendo los subsistema de individuo, industria y gobierno.

La idea no es descartar o señalar lo expuesto por Bossel o Castellano, sino tratar de experimentar las posibilidades de establecer indicadores que permitan iniciar el conocimiento sobre los niveles de complejidad y la capacidad de resiliencia de la estructura económica venezolana. El presente estudio estima que la incorporación de nuevos indicadores, permitirá evaluar el grado de diversificación y su modularidad tecnológico, y ahí se podrá reflejar de una mejor forma el nivel de elasticidad del subsistema económico.

En la tabla 2, el lector podrá apreciar que los subsistemas están guiados por unos orientadores que buscan enfocar el problema que se debe atender. Por supuesto, para ello se deberá generar una serie de condiciones que requieren de medidas estratégicas y que representan el punto focal de la resiliencia. Si no se generan esas condiciones, difícilmente se activará el mecanismo deseado. Sin embargo, de darse las condiciones deseadas, los indicadores propuestos permitirán evaluar el grado de efectividad o deficiencia de las tecnópolis en la activación de la resiliencia tecnológica en las capacidades del sistema económico venezolano.

\section{Consideraciones finales}

Uno de los aspectos más relevantes en la modernidad es el dinamismo en la generación de conocimiento, para obtener capacidades competitivas que le permitan a la sociedad global ser cada vez más productiva. Por supuesto, esa evolución del conocimiento ha estado enmarcada en la difusión del know-how dado por la experiencia, el pensamiento y/o el resultado de la combinación de ambos factores.

El desarrollo industrial de la globalización ha sido producto de los constantes procesos intensivos en mano de obra y de alto nivel de calificación. Por lo que el conocimiento pasa a ser el capital, expresado en talento humano y técnico de cualquier agente económico en la sociedad de hoy. 
Tabla 2. Posible impacto de las Tecnópolis en las capacidades del sistema económico venezolano

\begin{tabular}{|c|c|c|c|c|}
\hline \multicolumn{5}{|c|}{ VARIABLES E INDICADORES } \\
\hline Subsistemas & Orientadores & Estrategia & Indicadores p & las tecnópolis \\
\hline \multirow{4}{*}{ INDIVIDUO } & Productividad & \multirow{4}{*}{$\begin{array}{l}\text { Gestión del } \\
\text { conocimiento }\end{array}$} & Utilidad & Cantidad \\
\hline & Empleo & & $\begin{array}{c}\text { Puestos de } \\
\text { Trabajo }\end{array}$ & $\begin{array}{c}\text { Condiciones } \\
\text { favorables }\end{array}$ \\
\hline & $\begin{array}{l}\text { Capacitación } \\
\text { técnica }\end{array}$ & & $\begin{array}{l}\text { Formación } \\
\text { académica }\end{array}$ & $\begin{array}{c}\text { Formación en el } \\
\text { oficio }\end{array}$ \\
\hline & Habilidad tácita & & Experiencia & Necesidades \\
\hline \multirow[b]{4}{*}{ INDUSTRIA } & Producción & \multirow[b]{4}{*}{$\begin{array}{c}\text { Libre } \\
\text { desarrollo } \\
\text { comercial }\end{array}$} & Calidad & Cantidad \\
\hline & Sectores productivos & & Amplio & Estrecho \\
\hline & Diversificación & & Complementaria & Diversa \\
\hline & $\begin{array}{l}\text { Investigación y } \\
\text { Desarrollo }\end{array}$ & & Innovación & Imitación \\
\hline \multirow{4}{*}{ GOBIERNO } & Políticas de empleo & \multirow{4}{*}{$\begin{array}{l}\text { Instituciones } \\
\text { Inclusivas }\end{array}$} & Universal & Focalizada \\
\hline & $\begin{array}{c}\text { Libertad } \\
\text { económica }\end{array}$ & & $\begin{array}{c}\text { Sistema } \\
\text { cambiario }\end{array}$ & $\begin{array}{c}\text { Medidas } \\
\text { arancelaria }\end{array}$ \\
\hline & Infraestructura & & Financiamiento & Planificada \\
\hline & Seguridad & & Jurídica & Civil \\
\hline
\end{tabular}

Joseph Schumpeter argumentó que la virtud central de la economía de mercado era su capacidad para innovar, gracias a la competencia dentro del mercado. Sin embargo, el proceso de innovación no nace de la nada, sino que requiere de ciertos incentivos y demandas particulares que necesitan del aprendizaje y de la extensión del conocimiento de otros mercados. Para que se cumpla este proceso de generación de conocimiento, se presenta la necesidad de innovar y/o por el aprendizaje mediante la transferencia tecnológica.

North (1993) explica que las instituciones económicas inclusivas preparan el camino para dos motores de prosperidad: la tecnología y la educación. El desarrollo económico sostenido, casi siempre, va acompañado de mejoras tecnológicas que permiten que las personas (mano de obra), las tierras y el capital existente (edificios, maquinaria, etc.) pasen a ser más productivos. La educación y las habilidades de los trabajadores son los que generan el conocimiento científico sobre el que se construye el progreso y lo que permite la adaptación y adopción de estas tecnologías en varias líneas de negocio. De tal manera, que este aspecto es clave para comprender el papel que deben jugar las instituciones de las economías menos desarrolladas para ser más inclusivas, creando así organizaciones y/o estructuras que fomenten el cambio institucional mediante la gestión del conocimiento, por lo tanto, es evidente la pertinencia de las tecnópolis como una estrategia factible para activar la resiliencia tecnológica de un país.

En el caso de Venezuela, como nación mono exportadora de petróleo, sólo tiene la capacidad de actuar conforme a la dinámica del precio de este rubro. Su limitada capacidad de generación de investigación y desarrollo se refleja en la productividad de su estructura económica, lo que se expresa en una carente industrialización y baja competitividad en el sistema internacional. Por ello, se requieren generar estímulos para activar la resiliencia tecnológica del país, para así poder enfrentar los desafíos de la globalización. Es 
por ello que, la creación de tecnópolis sería una estrategia que generaría una causación circular positiva para mejorar la capacidad de respuesta ante los avatares de la economía mundial.

Adicionalmente, la conformación de tecnópolis requiere del establecimiento de indicadores para medir su impacto en el desarrollo sostenible de un país. De esta forma y en base de la propuesta de Bossel (1999) y Castellano (2013), se incorporaron nuevos indicadores que permiten evaluar el grado de diversificación y su modularidad tecnológica y, ahí, se podrá reflejar de una mejor forma el nivel de elasticidad del sistema económico. En base de los orientadores, cada subsistema requiere de una estrategia específica, como lo es para el individuo la gestión del conocimiento, para la industria el libre desarrollo comercial y, para el gobierno, la generación de instituciones inclusivas.

Esta breve propuesta, permitirá apoyar en la planificación estratégica del sistema económico venezolano, entendiendo que las relaciones económicas internacionales son interdependientes y lo global predomina sobre el mercado interno; por ello se necesitan estructuras que permitan que el Estado(gobierno) garantice que, tanto las universidades como el sector industrial cuenten con las condiciones necesarias para establecer las bases de un parque industrial competitivo dentro de la economía global.

\section{REFERENCIAS BIBLIOGRÁFICAS}

Acemoglu, D. y Robinson, J. (2003). Por qué fracasan los países. Deusto s.a. Ediciones. Consulta on-line en https://federalismoygobernabilidad.files.wordpress.com/2015/11/acemoglu.pdf [Recuperado, mayo 18]

Andrés, E. (2011). La Teoría de la complejidad y el caos en la ciencia regional. México: Instituto Tecnológico de OAXACA.

Bossel, H. Indicadores para el Desarrollo Sostenible: teoría, método y aplicaciones. Un Informe al Grupo Balaton. IISD International Institute for Sustainable Development. Recuperado de http://iisd.ca

Castellano, H. (2013). Caos y planificación del desarrollo. Caracas: CENDES - Universidad Central de Venezuela.

Castellano, H. (2014). Caos, resiliencia y gobernabilidad. Zulia: Universidad del Zulia. Multiciencias, Vol 14, $\mathrm{N}^{\circ} 4,(\mathrm{p}, 389-393)$.

Castells, M. y Hall, P. (1994). Las tecnópolis del mundo. La formación de los complejos industriales del siglo XXI. Madrid: Alianza Editorial.

De Mattos, C. (1996). Parques tecnológicos, medios de innovación y crecimiento nacional, regional y local. Reflexiones a partir de la experiencia francesa. Santiago de Chile: Pontificia Universidad Católica de Chile.

Held, D. McGrew, A. y otros (2002). Transformaciones Globales. Política, Economía y Cultura. México: Oxford University Press.

Hernández, R. (1998). Bases para un modelo de desarrollo Tecnológico, Industrial, Regional. Buenos Aires: Ediciones Macchi-UNSAM.

Mendoza, J. Jaimes, E. y otros (2008). Gestión empresarial promotora de Tecnopolos: Empresa Regional Sistema Hidráulico Trujillano. Revista Venezolana de Gerencia. Zulia: Universidad del Zulia (LUZ). Año 13. $N^{\circ} 41 .(75-94)$

Miguel, A. (Coord)(2011). La Teoría de la Complejidad y el Caos en la Ciencia Regional. México: Primera Edición. OAXACA.

Naim, M. (2014). El Fin del Poder. Caracas: Editorial Melvin, Libros El Nacional.

NORTH, D. (1993). Instituciones, cambio institucional y desempeño económico. México: Fondo de Cultura Económica.

Peraza, R. (1997). Estrategias para la Reestructuración Económica de Venezuela. Tesis para optar al título de Doctor en Ciencias Sociales. Tomo II. Caracas: Universidad Central de Venezuela.

Pérez, C. (1998). Innovaciones sociopolíticas para enfrentar los nuevos desafíos empresariales y sociales. Caracas: Ediciones Eureka. 
Pepe, E. (2008). Capital tecnológico. Entre socialismo y liberalismo, una visión macroeconómica y geopolítica del modo de producir. Serie Manuales Universitarios. Caracas: El Nacional-UCV.

Porter Michael, E. (2003). Ser Competitivo. Nuevas Aportaciones y Conclusiones. Barcelona: Deusto.

Sabato, J. y Botana, N. (1970). La ciencia y la tecnología en el desarrollo futuro de América Latina. Recuperado de https://books.google.co.ve/books

Santamonte, G. (2008). Las tecnopolis en las ciudades globales: El caso de la Ciudad de México. Tesis para obtener el grado de Maestro en Ciencias en Medio Ambiente y Desarrollo Integrado. México, D.F: Instituto Politécnico Nacional. Secretaria de Investigación y Postgrado. Estudios sobre medio ambiente y desarrollo integrado.

Sen, A. (2000). Desarrollo y Libertad. Colombia: Planeta.

Spolidoro, R. (1998). The Paradigm transition theory. A tool for guiding technopolitan trasnformations. En Delivering Innovation, Key Lessons from the World-Wide Network of Science and Technology Parks. Málaga: IASP.

Stewart, F. (1983). Tecnología y subdesarrollo. México: Fondo de Cultura Económica.

Solleiro y Castañon (Comp) (2008). Gestión Tecnológica: Conceptos y prácticas. México: Programa Iberoamericano de Ciencia y Tecnología para el Desarrollo (CYTED).

Touraine, A. (2005). Un nuevo paradigma para comprender el mundo de hoy. Barcelona - España: Paidós. 